\title{
Efectos psicológicos de la cuarentena
}

\author{
Psychological effects of quarantine
}

\section{Comentado de:}

Brooks SK et al, Lancet 2020;395(10227):912-920. PMID: $32112714^{1}$

\section{Objetivo}

Conocer el impacto psicológico de la cuarentena para explorar sus posibles efectos sobre la salud mental y el bienestar psicológico, y los factores que contribuyen o los mitigan, para generar una síntesis rápida de la evidencia con el objeto de formular políticas públicas.

\section{Diseño}

Revisión rápida de evidencia.

\section{Fuente de datos}

Se realizaron búsquedas en MEDLINE, PsycINFO, y Web of Science con una combinación de términos relacionados con la cuarentena (por ejemplo, 'cuarentena' y 'paciente aislamiento') y resultados psicológicos (por ejemplo, 'psicológico' y 'estigma').

\section{Selección de estudios}

Se incluyeron 24 estudios de 3.166 registros recuperados por la estrategia de búsqueda, que fueron investigaciones primarias publicadas en revistas revisadas por pares en inglés o italiano. La población de interés estuvo conformada por población general, personal de hospital, universitarios y escolares que hayan realizado cuarentena fuera del hospital al menos 24 horas. A su vez, los estudios debían incluir información acerca de las enfermedades mentales o el bienestar psicológico de los participantes.

\section{Métodos}

Se seleccionaron 24 estudios entre los 3.166 artículos recuperados por la estrategia de búsqueda, los cuales se realizaron en diez países e incluyeron personas con diagnóstico, sospecha o exposición a SARS (11 estudios), Ébola (cinco estudios), influenza H1N1 2009 y 2010 (tres estudios), síndrome respiratorio del Medio Oriente (dos estudios) e influenza equina (un estudio). Uno de estos estudios se relacionaba con H1N1 y SARS.

Respecto a las herramientas de medición, diez estudios utilizaron pruebas específicas (cuestionarios IES-R para evaluar síntomas de estrés postraumático, CES-D para evaluar la depresión, GAD-7 para evaluar la ansiedad, SARS MBI-GS para evaluar el agotamiento, STAXI-2 para evaluar la ira, GHQ-30 para evaluar trastornos psicológicos, etc.), nueve estudios realizaron entrevistas en profundidad, y el resto implementó encuestas o preguntas específicas.

\section{Resultados}

La mayoría de los estudios informaron efectos psicológicos negativos como estrés postraumático, confusión y enojo, y factores estresantes como duración de la cuarentena, temor a infección, frustración, aburrimiento, suministros e información inadecuados, dificultades económicas y estigma social.

En un número reducido de estudios, el impacto psicológico de la cuarentena pudo detectarse meses o años después. A su vez, el factor demográfico no tuvo relación con resultados psicológicos negativos.

Si bien en los trabajadores de la salud, se observó alta prevalencia de angustia psicológica, no hubo una diferencia significativa respecto a trabajadores no sanitarios. Sin embargo, en unos pocos estudios se observó que ser personal de salud se relacionaba con síntomas más severos.

Algunas intervenciones que se sugieren para mitigar los efectos psicológicos incluyen: mantener la cuarentena la menor cantidad de tiempo posible; brindar a la población tanta información como sea posible; proporcionar suministros adecuados; reducir el aburrimiento y mejorar la comunicación; priorizar el altruismo a la compulsión (es decir una cuarentena voluntaria y no mandatoria).

\section{Conclusiones}

Ante la implementación de medidas sanitarias como la cuarentena, se debería garantizar que esta experiencia sea lo más tolerable posible, considerando proporcionar una justificación clara de la necesidad de aplicarla, los motivos de su duración, información sobre protocolos de cuidados y la provisión de suministros básicos. Al mismo tiempo, se deberían ofrecer actividades adecuadas para la población y apelar al altruismo, recordando los beneficios de la cuarentena como un bien público.

Fuentes de financiamiento/Conflicto de interés de los autores: National Institute for Health Research, King's College London, Public Health England, University of East Anglia y Newcastle University. Los autores declararon no tener conflictos de interés.

\section{Comentario}

Algunas de las limitaciones que detectamos en esta síntesis de evidencia, por tratarse de una revisión rápida, radica en que la búsqueda de estudios se limitó a publicaciones evaluadas por pares, no se exploró literatura gris potencialmente relevante y en que los estudios incluidos no fueron sometidos a una evaluación de calidad. Por otro lado, los resultados surgen de pequeños grupos de personas, durante períodos variables (entre 3 y 30 días como máximo), y, a su vez, los tamaños muestrales fueron por lo general pequeños. Pocos estudios compararon a los participantes que realizaron cuarentena con aquellos que no recibieron aislamiento, y sólo un estudio efectuó un seguimiento de los participantes a lo largo del tiempo. Finalmente es importante destacar que la heterogeneidad en las herramientas de medición utilizadas en los diversos estudios dificulta las comparaciones.

En Argentina, en marzo de 2020, se realizó el "Relevamiento del impacto social de la cuarentena", con el objetivo de predecir el potencial impacto psicosocial del aislamiento social preventivo y obligatorio en el contexto de la pandemia de COVID-19 en los barrios vulnerables. Una minoría de participantes expresó que la información recibida en los barrios era insuficiente, que había sobreinformación o noticias falsas, y que la información fue inadecuada por la dificultad de aplicación ante la deficiencia de infraestructura y la falta de insumos de limpieza, con mayor grado de afectación en las personas en situación de calle. También se registró pérdida de las actividades laborales en el sector informal y en la economía popular, lo que se 
asoció con la disminución de la posibilidad de abastecerse, la sobrecarga de las redes de distribución de alimentos (estatales, organizaciones, pequeños comerciantes) y la suba de precios. A su vez, se identificaron dificultades para el acceso a los medicamentos y la continuidad de los tratamientos médicos prescritos, la falta de elementos de protección para el personal de salud, comedores y fuerzas de seguridad. Por otro lado, se percibió un aumento de la violencia familiar y vecinal, en relación a la pérdida de contención social cotidiana en los espacios comunitarios ${ }^{2}$.

Una revisión sistemática que evaluó las consecuencias sociales de la cuarentena determinó que la angustia psicológica, el aumento de las desigualdades en la comunicación, la inseguridad alimentaria, los desafíos económicos, la disminución del acceso a la atención médica, la prestación alternativa de educación y la violencia de género se identificaron como consecuencias sociales negativas de la cuarentena comunitaria en seis epidemias de enfermedades infecciosas, incluida la actual, por COVID-19. Por el contrario, una consecuencia positiva durante las cuarentenas anteriores fueron las actitudes altruistas. Las políticas de cada país fueron muy variadas en cuanto a la forma en que se abordaban las necesidades de las personas afectadas, especialmente las de mayor marginación social estaban socialmente marginadas ${ }^{3}$.

Otro estudio que abordó el impacto psicológico de la cuarentena documentó que, en comparación con los controles, las personas que experimentaron el aislamiento preventivo o la cuarentena tenían un mayor riesgo de resultados adversos de salud mental, particularmente después de una duración de la misma de 1 semana o más ${ }^{4}$. En el caso de los trastornos depresivos, la razón de probabilidad (odds Ratio [OR]) fue 2,79, con intervalo de confianza (IC) del $95 \%$ entre 1,47 y 5,32; para los trastornos de ansiedad el OR fue 2,0 (IC $95 \%$ 0,88 a 4,53) y para los trastornos relacionados con el estrés el OR fue 2,74 (IC $95 \% 1,49$ a 5,03).

Un revisión sistemática y metaanálisis que sintetizó estudios llevados a cabo en niños y adolescentes encontró que el 34,5\%, $41,7 \%, 42,3 \%$ y $30,8 \%$ de los niños sufrían ansiedad, depresión, irritabilidad y falta de atención, respectivamente. Del total de niños evaluados, $79,4 \%$ se vio afectado negativamente por la pandemia y la cuarentena; al menos $22,5 \%$ de los niños tenía un miedo significativo al COVID-19, mientras que 35,2\% tenía aburrimiento y 21,3\%, alteraciones del sueño. En sintonía con estos resultados, el 52,3\% y el 27,4\% de los cuidadores desarrollaron ansiedad y depresión, respectivamente, mientras estaban aislados con los niños ${ }^{5}$.

\section{Conclusiones de las comentadoras}

En contexto de la pandemia por COVID-19 y teniendo en cuenta sus potenciales consecuencias en la salud mental de las personas de las medidas de confinamiento, esta revisión rápida resultó oportuna en el momento en el cual se realizó, e investigaciones posteriores confirmaron sus hallazgos.

De cara a la posibilidad de nuevas cuarentenas y restricciones dispuestas para el control de la pandemia, es fundamental recordar que los formuladores de políticas públicas deben equilibrar los pros y los contras de las las medidas restrictivas a la libertad de circulación de las personas, brindar una orientación clara y coherente a la población acerca de las mismas, promover acciones multisectoriales para abordar las desigualdades sociales y mitigar el impacto negativo de los cierres por COVID-19, al mismo tiempo que fortalecer y trabajar sobre estrategias efectivas para disminuir la carga de la enfermedad, como las campañas de vacunación.

Natalia Jimena Ponce de León [ Servicio de Medicina Familiar y Comunitaria, Hospital Italiano de Buenos Aires. natalia.poncedeleon@hospitalitaliano. org.ar ]

Carolina Carrara [ Servicio de Medicina Familiar y Comunitaria, Hospital Italiano de Buenos Aires; Departamento de Salud Pública, Instituto Universitario Hospital Italiano de Buenos Aires. carolina.carrara@hospitalitaliano.org.ar ]

Ponce de León NJ, Carrara C. Efectos psicológicos de la cuarentena. Evid Actual Pract Ambul. 2021;24(2):e002123. Available from: https://dx.doi.org/ 10.51987/EVIDENCIA.V24I3.6947. Comentado de: Brooks SK, et al. The psychological impact of quarantine and how to reduce it: rapid review of the evidence. Lancet 2020;395(10227):912-920. PMID: 32112714

\section{Referencias}

1. Brooks SK, Webster RK, Smith LE, et al. The psychological impact of quarantine and how to reduce it: rapid review of the evidence. The Lancet. 2020;395(10227):912-920. Available from: 10.1016/s0140-6736(20)30460-8;https://dx.doi.org/10.1016/s0140-6736(20)30460-8.

2. Etchevers MJ, Garay CJ, Putrino NI, et al. Argentinian Mental Health During the COVID-19 Pandemic: A Screening Study of the General Population During Two Periods of Quarantine. Clinical Psychology in Europe. 2021;3(1):e4519. Available from: 10.32872/cpe.4519;https://cpe.psychopen.eu/ index.php/cpe/article/view/4519/4519.html.

3. Chu IY, Alam P, Larson HJ, et al. Social consequences of mass quarantine during epidemics: a systematic review with implications for the COVID-19 response. J Travel Med. 2020;27(7):192-192. Available from: 10.1093/jtm/taaa192.

4. Henssler J, Stock F, van Bohemen J, et al. Mental health effects of infection containment strategies: quarantine and isolation-a systematic review and meta-analysis. Eur Arch Psychiatry Clin Neurosci. 2021;271(2):223-234. Available from: 10.1007/s00406-020-01196-x;https://dx.doi.org/10. 1007/s00406-020-01196-x.

5. Panda PK, Gupta J, Chowdhury SR, et al. Psychological and Behavioral Impact of Lockdown and Quarantine Measures for COVID-19 Pandemic on Children, Adolescents and Caregivers: A Systematic Review and Meta-Analysis. J Trop Pediatr. 2021;67(1):122-122. Available from: 10.1093/ tropej/fmaa122;https://dx.doi.org/10.1093/tropej/fmaa122. 